\title{
Performance of LTE Linear MIMO Detectors: Achievable Data Rates and Complexity
}

\author{
Dragan Samardzija, Milos Pilipovic, Dusica Marijan, Jaroslav Farkas, Miodrag Temerinac \\ University of Novi Sad \\ Novi Sad, Serbia \\ dragan.samardzija, milos.pilipovic, dusica.marijan, jaroslav.farkas, miodrag.temerinac @rrt-rk.com
}

\begin{abstract}
In this study we analyze performance of linear single user multiple-input multiple-output (SU-MIMO) detector applied in 3GPP LTE wireless systems. We consider minimum mean square error (MMSE) linear detectors based on (i) explicit matrix inversion, and (ii) adaptive gradient algorithm. We present the average achievable data rates as a function of different mobile terminal speeds, using the Jakes model, with symbol-by-symbol temporal variations. Mean and cell-edge rates are determined using two-dimensional multi-cell model. The LTE-specific reference signal (i.e., pilot) arrangements are considered. In addition, the implementation complexity is analyzed. At the expense of higher implementation complexity, for higher mobile terminal speeds and signal-to-interference-andnoise ratio (SINR) the detector based on explicit matrix inversion outperforms the one based on the adaptive gradient algorithm. Furthermore, we show that a corresponding single-input multiple-output (SIMO) solution outperforms MIMO for moderate and high mobile terminal speeds. In addition, complexity-wise the SIMO transmission is shown to be more efficient. The results indicate that the MIMO transmission with linear detection should be applied only in a very limited number of channel conditions: (i) high SINR, and (ii) low mobility. This study may be used as a basis for establishing a trade-off between the data rates, complexity and multiple antenna arrangements.
\end{abstract}

Keywords-LTE; MIMO; SIMO;

\section{INTRODUCTION}

Long Term Evolution (LTE) is recognized as the leading future cellular technology $[1,2]$. It is specified in by 3 GPP in Release 8, with future releases aimed to further improve its performance. The LTE technology is optimized for high-speed packed data transfer, with the physical layer based on OFDMA $[3,4]$. LTE specifies a number of multiple antenna techniques that are considered as the key enablers of the high capacity and/or improved coverage transmission. The application of a particular multiple antenna technique is adaptively determined based on the radio propagation conditions and specific application requirements. In addition, LTE is to complement the existing $2 \mathrm{G}, 3 \mathrm{G}$ and WLAN technologies [3].

In this study we focus on multiple antenna techniques as the most demanding implementation problem affecting performance, cost and power consumption of the base stations and mobile terminals. This study may be used as a basis for

This work was partially supported by the Ministry of Science and Technological Development of Republic Serbia under the project No. 11005, year 2008 . establishing a trade-off between data rates, complexity and multiple antenna arrangements.

In Section II we present an overview of multiple antenna techniques that are applied in LTE. In Section III we describe the minimum mean square error (MMSE) linear multiple-input multiple-output (MIMO) detectors based on (i) explicit matrix inversion, and (ii) adaptive gradient algorithm. In Section IV, we present the achievable data rates as a function of different mobile terminal speeds, using the Jakes model, with symbolby-symbol temporal variations. Mean and cell-edge rates are presented using two-dimensional multi-cell model. The LTEspecific reference signal (i.e., pilot) arrangements are considered. In Section V the implementation complexity is analyzed.

At the expense of higher implementation complexity, for higher mobile terminal speeds and signal-to-interference-andnoise ratio (SINR) the detector based on explicit matrix inversion outperforms the one based on the adaptive gradient algorithm. Furthermore, we show that the corresponding single-input multiple-output (SIMO) solution outperforms MIMO for higher mobile terminal speeds, with a significantly lower implementation complexity.

\section{LTE MUltiple ANTENNA TECHNIQUES OVERVIEW}

An overview of the LTE multiple antenna techniques is presented in this section In general, a greater number of antennas result in improved performance in terms of throughput and coverage, nevertheless, directly affecting the cost of the base station and mobile terminal implementation and their power consumption.

Note that in the LTE nomenclature, eNB corresponds to base station, while UE (user equipment) to mobile terminal.

\section{A. Receiver Antenna Diversity}

Receiver antenna diversity may be applied both on the base station and terminal side. The diversity is to help receiver improve SINR through increased received power, and ability to suppress detrimental effects of small-scale fading and interference.

In the case of the receiver diversity, there is no limit on the number of antennas that may be applied. However, the number of base station antennas per sector is expected not to exceed 4 
(in the future 8). The number of mobile terminal antennas is expected not to exceed 4 .

\section{B. Transmit Antenna Diversity}

During the transmit antenna diversity transmission the following precoding operation is performed by the base station [5].

$$
\left[\begin{array}{c}
y(1) \\
\cdots \\
y\left(N_{T X}\right)
\end{array}\right]=\mathbf{W}\left[\begin{array}{c}
x(1) \\
\cdots \\
x(L)
\end{array}\right]
$$

where $N_{T X}$ is the number of base station antennas and $L$ is the number of layers, i.e., spatial data streams sent by the base station to the mobile terminal. Furthermore, the vector $\mathbf{x}=[x(1)$ $\ldots x(L)]^{\mathrm{T}}$ corresponds to the sent data streams, and the vector $\mathbf{y}$ $=\left[y(1) \ldots y\left(N_{T X}\right)\right]^{\mathrm{T}}$ to the signal transmitted over the $N_{T X}$ antennas. $\mathbf{W}$ is the spatial precoding matrix, which is defined by the 3GPP specification [5]. Specifically, LTE base station may apply one of many possible spatial precoders depending on the number of antennas. The base station decides on $\mathbf{W}$, and a mobile terminal is informed about it via a control channel. The goal of the transmit antenna diversity is primarily to improve the coverage, i.e., reliability of data transfer through spatial and frequency diversity. It is considered to be less demanding that the spatial multiplexing therefore will not be a subject of further analysis in this study.

\section{Spatial Multiplexing}

Spatial multiplexing is also known as MIMO. In LTE, spatial multiplexing is specified either as single user (SUMIMO) or multi-user (MU-MIMO). In general, the transmitted signal is generated as in (1), where the spatial precoder $\mathbf{W}$, and the number of layers and antennas are set according to either SU- or MU-MIMO. It is up to the base station to decide whether and how any of the techniques is applied. The layers occupy the same time and frequency resources realizing spatial multiplexing, i.e., spatial reuse which is unique for MIMO resulting in improved capacity, i.e., throughput.

SU-MIMO is available on the downlink and it requires multiple antennas both on the base station as well as on the mobile terminal side. In LTE, number of base station antennas is expected to be either 2 or 4 , while mobile terminal antennas 1,2 or 4 . Minimum number of antennas on both ends determines the maximum number of layers $L$, i.e., streams that could be transmitted.

In the closed-loop scenarios, the spatial precoder $\mathbf{W}$ in (1), is selected based on the mobile terminal feedback, indicating possible rank of the channel and/or deciding on the exact codebook matrix W. In the open-loop case, the base station implicitly decides on $\mathbf{W}$. In either case, the mobile terminal will be informed about the applied spatial precoder $\mathbf{W}$.

SU-MIMO is considered to be the most demanding in terms on the mobile terminal complexity and cost. The goal of the mobile terminal MIMO receiver is to estimate the vector $\mathbf{x}$ in (1). In further text, SU-MIMO will be a subject of detailed implementation analysis.
MU-MIMO is defined both on the uplink and downlink. In this case layers are dedicated to or originate from different mobile terminals. Considering the mobile terminal complexity and cost, MU-MIMO is considered less demanding than SUMIMO, thus it will not be further analyzed.

\section{Transmitter Beamforming}

Transmitter beamforming is applied on the downlink, and only one layer is transmitted over $N_{T X}$ antennas, where $N_{R X}$ may be 2,4 , or even 8 .

In general, the transmitted signal is generated as in the expression (1). The spatial precoder $\mathbf{W}$ may be selected from the standard-specific code-book, in which case mobile terminal is informed about the selection via a control channel. Alternatively $\mathbf{W}$ may be chosen arbitrary, in which case dedicated mobile terminal pilot symbols are subject of the precoding, together with the data-carrying signal $\mathbf{x}$.

The transmitter beamforming is primarily indented to improve the received signal strength, and thus improve the system reliability and coverage. The burden of the processing is on the base station side, with a little demand on the mobile terminal. Consequently, this technique will not be a subject of further study in this document.

\section{SU-MIMO DETECTION}

SU-MIMO detection is the most demanding physical layer operation on the mobile terminal side. Many different MIMO detection schemes could be applied, broadly classified as linear, and non-linear detection algorithms [6].

In general, linear MIMO schemes are less complex, with low and predictable latency. On contrary, non-linear schemes are typically more complex, with longer latencies especially if successive inter-layer cancellation is applied in conjunction with the channel decoding. However, non-linear schemes are expected to have a better performance in terms of higher throughput (i.e., closer to the ultimate theoretical limits).

Considering lower complexity, latency and analytic tractability in this study we consider a liner MIMO detection scheme based on the minimum mean square error criterion, i.e., MMSE SU-MIMO detector.

Per each OFDMA subcarrier, the received signal at the mobile terminal is

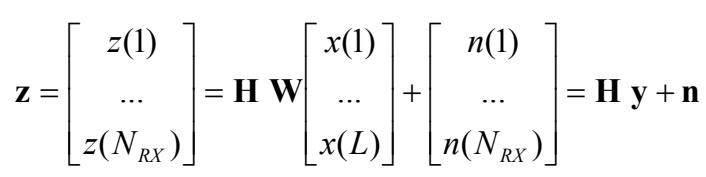

where $\mathbf{H}$ is $N_{R X} \mathbf{x} N_{T X}$ MIMO channel between $N_{T X}$ base station and $N_{R X}$ mobile terminal antennas. The vector $\mathbf{n}=[n(1) \ldots$ $\left.n\left(N_{R X}\right)\right]^{\mathrm{T}}$ is the noise-plus-interference vector at the receive antenna array. In addition, the vector $\mathbf{y}$ is defined in (1). The linear MIMO detection performs the following operation,

$$
\underline{\mathbf{y}}=\mathbf{Q} \mathbf{z}
$$


where $N_{T X} \mathbf{X} N_{R X}$ matrix $\mathbf{Q}$ is determined by the receiver, and applied to obtain the estimate of the transmitted vector $\mathbf{y}$. The above detection is performed per each OFDMA subcarrier, where all the components of the vector $\mathbf{z}$ correspond to one subcarrier, received by different mobile terminal antennas. After the estimate $\mathbf{y}$ is obtained, the transmitted data-carrying vector estimate is

$$
\underline{\mathbf{x}}=\mathbf{W}^{-1} \underline{\mathbf{y}}
$$

where $\mathbf{W}$ is the precoder in (1). Furthermore, the matrix $\mathbf{Q}$ is the MMSE linear detector defined as,

$$
\mathbf{Q}=\left(\underline{\mathbf{H}}^{\mathrm{H}} \underline{\mathbf{H}}+\underline{\mathbf{I}}_{\mathbf{f}}\right)^{-1} \underline{\mathbf{H}}^{\mathrm{H}}
$$

where $\underline{\mathbf{H}}$ and $\underline{\mathbf{I}}_{\mathbf{f}}$ are estimates of the MIMO channel and interference covariance matrix, respectively [6].

Based on the above, the MIMO detector at the mobile terminal performs the following steps.

1. The vector $\mathbf{z}$ is received where each component corresponds to the same OFDMA subcarrier and different receive antenna. The subcarrier demapping is performed before the MIMO detection.

2. Whenever a new estimate of the MIMO channel $\underline{\mathbf{H}}$ or interference covariance $\underline{\mathbf{I}}_{\mathbf{f}}$ is obtained, the MMSE detector has to be calculated as given in the expression (5). This step may be implicit to the channel and interference estimation, and performed less frequently than the rest of the MIMO detection procedure. The corresponding analysis will be presented further in the text.

3. The estimate of the transmitted vector $\mathbf{y}$ is obtained as given in the expression (3).

4. The estimate of the data-carrying vector $\mathbf{x}$ is obtained as given in the expression (4).

Note that the detection in (3) and detector in (5) may be used for different number of receive $N_{R X}$ and transmit $N_{T X}$ antennas. For example, in the SIMO case, the detector $\mathbf{Q}$ in (5) is a $1 \times N_{R X}$ row vector.

On the downlink, in order to enable coherent reception, base station transmits pilot symbols, i.e., reference signals (RSs). The reference signals occupy specific time interval and frequency (subcarrier) locations, i.e., resource elements. The locations are specified within a block of subcarriers and interval allocated to a specific mobile terminal. This block is known as physical resource block (PRB) and consists of 12 subcarriers and 6 or 7 symbols, for extended or normal cyclic prefix, respectively. In the case of multiple antenna transmission, unique resource elements are reserved for the $\mathrm{RSs}$, enabling mobile terminal to estimate the MIMO channel $\mathbf{H}$ for the given PRB. The remaining resource elements are used to carry data, and will be the subject of the detection in (3) and (4).

\section{A. MMSE Detector Calculation - Matrix Inversion}

In the case of the MMSE SU-MIMO detection, one option is to explicitly estimate the channel $\mathbf{H}$ and interference covariance matrix $\mathbf{I}_{\mathbf{f}}$, and then determine the detector as given in (5). The estimates of $\mathbf{H}$ and $\mathbf{I}_{\mathbf{f}}$ are obtained using the received reference signals. Because of the explicit matrix inversion in (5) this approach is demanding in terms of the implementation complexity. Details of an algorithm for explicit matrix inversion are given in the following, for $N_{R X}=4$ [7].

TABLE I. PSEUDO CODE FOR THE MMSE SU-MIMO DETECTOR USING

\begin{tabular}{|c|c|}
\hline Algorithm & Notes \\
\hline 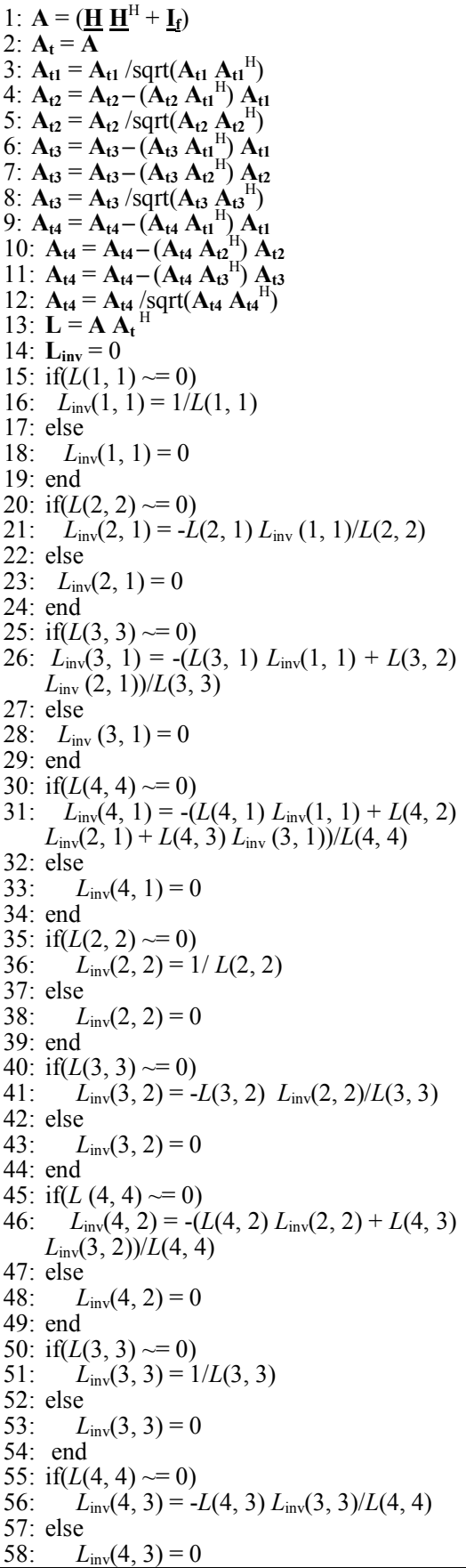 & 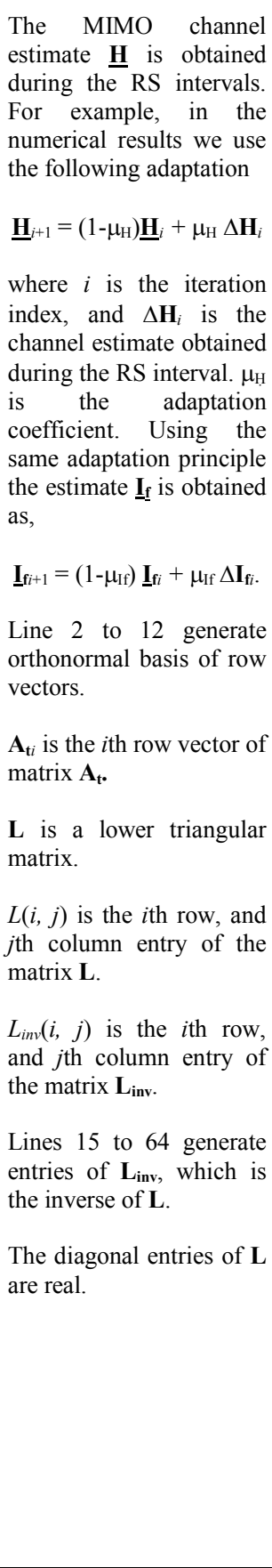 \\
\hline
\end{tabular}
EXPLICIT MATRIX INVERSION. 


\begin{tabular}{l} 
59: end \\
60: if $(L(4,4) \sim=0)$ \\
61: $L_{\text {inv }}(4,4)=1 / L(4,4)$ \\
62: else \\
63: $L_{\text {inv }}(4,4)=0$ \\
64: end \\
65: $\mathbf{A}_{\text {inv }}=\mathbf{A}_{\mathbf{t}}^{\mathrm{H}} \mathbf{L}_{\text {inv }}$ \\
66: $\mathbf{Q}=\underline{\mathbf{H}}^{\mathrm{H}} \mathbf{A}_{\text {inv }}$ \\
\hline
\end{tabular}

Strictly speaking, the detector $\mathbf{Q}$ in Table I, is derived only for the time interval and frequency that correspond to the position of the particular reference signals. Nevertheless, it is applied to all neighboring data-carrying resource elements to perform the detection in (3) and (4), and estimate the transmitted data. This implicitly assumes flat fading for all subcarriers in the vicinity of the subcarrier that carries the reference signal.

The algorithm in Table I is particularly suitable for a DSP or matrix co-processor implementation platform, that implements addition, multiplication, square-root and division as a set of basic arithmetic operations.

\section{B. MIMO Detector Calculation-Adaptive Gradient Algorithm}

In order to lower the implementation complexity in this study we also investigate an adaptive scheme that determines the detector $\mathbf{Q}$. The scheme implicitly (i) estimates the MIMO channel $\mathbf{H}$, and (ii) interference covariance $\mathbf{I}_{\mathbf{f}}$. It is an iterative gradient algorithm [8] defined in the following.

$$
\mathbf{Q}_{i+1}=\mathbf{Q}_{i}-\mu \mathbf{g}_{i}
$$

where $i$ is the iteration index, $\mu$ is the adaptation coefficient, and $\mathbf{g}_{i}$ is a gradient

$$
\mathbf{g}_{i}=-\left(\mathbf{y}_{i}-\mathbf{Q}_{i} \mathbf{z}_{i}\right) \mathbf{z}_{i}^{\mathrm{H}} .
$$

The vector $\mathbf{z}_{i}$ is the received vector corresponding to the particular time interval and subcarrier, i.e., resource element that carries reference signals. Since the reference signals are known at the mobile terminal, it can form the corresponding transmitted vector $\mathbf{y}_{i}$ and include it in the expression (7).

Strictly speaking, the detector in (6) is derived only for the time interval and frequency that correspond to the position of the particular reference signals. Nevertheless, it is applied to all neighboring data-carrying resource elements to perform the detection in (3) and (4), and estimate the transmitted data. This implicitly assumes flat fading for all subcarriers in the vicinity of the subcarrier that carries the reference signal.

Note that the adaptation coefficient $\mu$ is selected to achieve a trade-off between the noise sensitivity and speed of adaptation. The coefficient may be dynamically set so that the optimum is achieved.

\section{PERFORMANCE EVALUATION}

To evaluate performance of the presented detection schemes each element of the MIMO channel matrix $\mathbf{H}$ is modeled as an independent variable with the temporal evolution according to the Jakes model [9]. For example, for the time interval $k$, the channel between transmit antenna $m$ and receive $n$ (the $m$ th column and $n$th row element of $\mathbf{H}$ ) is given as

$$
h_{n, m}(k)=\frac{1}{\sqrt{N_{f}}} \sum_{i=0}^{N_{f}-1} e^{i\left(2 \pi \mathrm{f}_{\mathrm{d}} \cos \left(2 \pi i / N_{f}\right)(k-1) T_{s y m}+\varphi_{i}\right)}
$$

where $N_{f} \rightarrow \infty$ (number of components in the Jakes model), $f_{d}$ is the Doppler frequency

$$
f_{d}=\frac{v}{c} f_{c}
$$

where $v$ is the UE speed, $f_{c}=2 \mathrm{GHz}$ is the carrier frequency and $c$ is the speed of light. $T_{\text {sym }}=71.36$ usec is the duration of LTE symbol (for normal cyclic prefix). $\varphi_{\mathrm{I}}$ is initial random phase, drawn from a uniform distribution $U(0,2 \pi)$. The above model results in a symbol-by-symbol evolution of the MIMO channel H.

To assess the performance, the average noise power is determined after the detection. Namely, for the symbol interval $k$, and the given channel $\mathbf{H}(k)$ and detector $\mathbf{Q}(k)$, the postdetection noise power level is

$$
N_{p d}(k)=\mathrm{E}_{\mathbf{x}}\left[\frac{(\mathbf{x}-\mathbf{D}(k) \underline{\mathbf{x}})^{\mathrm{H}}(\mathbf{x}-\mathbf{D}(k) \underline{\mathbf{x}})}{N_{T X}}\right]
$$

where $\mathbf{D}(k)$ is a diagonal matrix, whose entries are inverses of the diagonal entries of matrix $\mathbf{Q}(k) \underline{\mathbf{H}}(k)$, and $\underline{\mathbf{H}}(k)$ is an estimate of $\mathbf{H}(k)$. Using the above post-detection noise variance, the achievable rate for interval $k$ is

$$
R(k)=\eta(k) N_{T X} \log _{2}\left(1+\frac{1}{N_{p d}(k)}\right)
$$

for $\mathrm{E}\left[\mathbf{x}^{\mathrm{H}} \mathbf{x}\right]=N_{T X}$. The coefficient $\eta(k)$ accounts for the RS overhead. For example, for the intervals that carry the RSs, $\eta(k)=8 / 12$ (because according to the RS arrangement defined in TS 36.211, out of the 12 available subcarriers per PRB, 4 are allocated to the RSs). In the case of the intervals that do not carry any $\mathrm{RS}, \eta(k)=1$. To achieve the above rate a channel coding should be applied across all the layers, with the postdetection noise being additive white Gaussian noise (AWGN). The rate averaged over all channel evolutions is

$$
R=\mathrm{E}_{\mathbf{H}(0)}\left[\lim _{K \rightarrow \infty} \frac{\sum_{k=0}^{K} R(k)}{K}\right] .
$$

In order to evaluate the multi-cell performance, SINR values are randomly generated according the distribution known as geometry of a reuse-1 multi-cell wireless system. It is obtained from simulations with the following parameters. Each sector and base station uses the same frequency channel (i.e., reuse-1 system). Each base station has three sectors $\left(120^{\circ}\right.$ sector). Path loss is with exponent -3.76 , shadowing variance 8 $\mathrm{dB}$ and base station shadowing correlation is 0.5 . The transmit power is set to achieve $20 \mathrm{~dB}$ SNR at the cell edge. Each SINR value accounts for large scale fading effects (path loss, shadowing and antenna pattern). Consequently, for each SINR 
value, an independent complex variable is generated modeling a small-scale fading between any transmit and receive antenna pair. The variable evolves according to the Jakes model in (8).

In Fig. 1 we present cumulative distribution function $(c d f)$ of rates in (12) for the 4x4 MIMO and 1x4 SIMO detectors, all for the mobile terminal speed $v=3 \mathrm{kmph}$. The rates correspond to the multi-cell wireless system model that is described above.

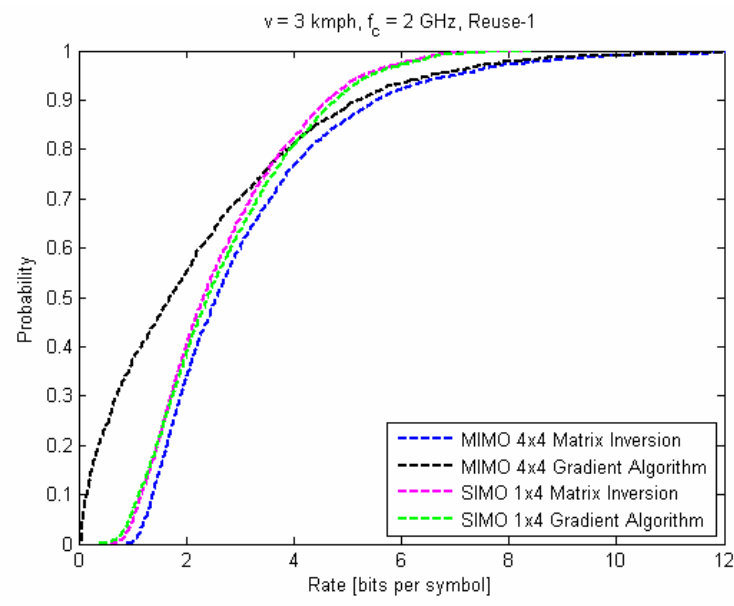

Figure 1. $\quad c d f$ of rates in reuse- 1 system, $\mathrm{v}=3 \mathrm{kmph}$.

In Fig. 2 and Fig. 3 we tabulate the mean and cell-edge rate, respectively. The cell-edge rate is defined as the 5-percentile rate (i.e., only $5 \%$ of rates are lower that the cell-edge rate). The rates are presented for different mobile terminal speeds: 3, 30 and $60 \mathrm{kmph}$. Based on the presented results, the MIMO transmission should be applied only at low mobile terminal speeds, with the MIMO detector using explicit matrix inversion. At moderate and high speeds, a single layer SIMO transmission should be applied, since it outperforms MIMO alternatives both in terms of mean and cell-edge rates. Furthermore, the MIMO detector based on the adaptive gradient algorithm performs poorly in terms of cell-edge rates.

\section{COMPLEXITY ANALYSIS}

In this analysis we will consider the following phases of the MIMO detection.

1. MIMO detector calculation, considering both the schemes based on

a. matrix inversion in (5) and Table I, as well as

b. adaptive gradient algorithm in (6) and (7).

2. Data detection, i.e., estimation of the received data in (3) and (4).

The complexity of the above schemes is presented in terms of the number of arithmetic operations needed (i.e., multiplications, additions, square root and divisions). We analyze the most demanding 4x4 MIMO LTE scenario where $N_{T X}=4$ and $N_{R X}=4$, and the detector calculation is performed six times per PRB (according to the RS arrangement for $N_{T X}=$ 4 defined in 3GPP TS 36.211). Furthermore, per each PRB there are 72 data-carrying symbols.

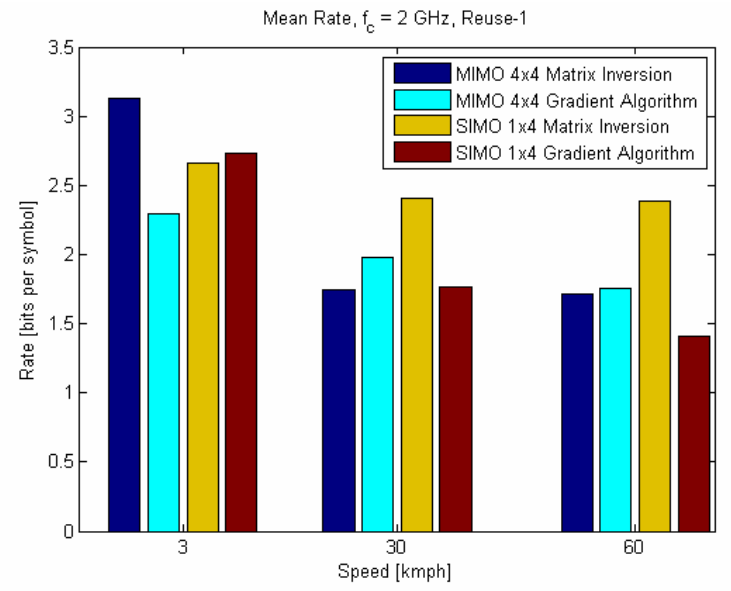

Figure 2. Mean rate as a function of mobile terminal speed.

In addition, complexity of the equivalent $1 \times 4$ SIMO detector is presented. The SIMO detector calculation is perform four times per PRB (according to the RS arrangement for $N_{T X}=1$ defined in 3GPP TS 36.211). In the SIMO case, per each PRB there are 80 data-carrying symbols, due to a lower number of the RSs compared to the MIMO case.

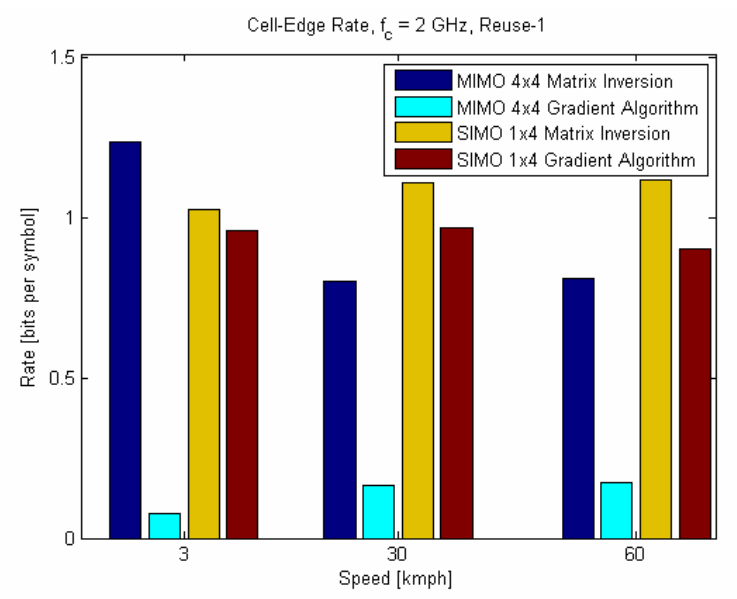

Figure 3. Cell-edge rate as a function of mobile terminal speed.

In Fig. 4 we present the total number of operations per second (total sum of multiplications, additions, square root and divisions) as a function of bandwidth allocated to UE. From the presented results, the version with the explicit matrix inversion is significantly more complex, but outperforms the adaptive gradient solution for higher speeds. As expected, the MIMO transmission is more demanding than the SIMO transmission.

To quantify relationship between the complexity and achievable data rates we tabulate the ratio between total number of operations versus mean rate, per PRB. Since the mean rate in Fig. 2 corresponds to one symbol, it is multiplied by the number of resource elements in PRB, i.e., $12 \times 7$. The ratio is presented in Fig. 5. From the results, the SIMO schemes are very effective because they require the lowest number of operations per transmitted information bit, on average. The SIMO detector based on the adaptive gradient 
algorithm is particularly effective, i.e., its ratio in Fig. 5 is the lowest. This analysis may be used as a basis for establishing a trade-off between data rates, complexity and multiple antenna arrangements.

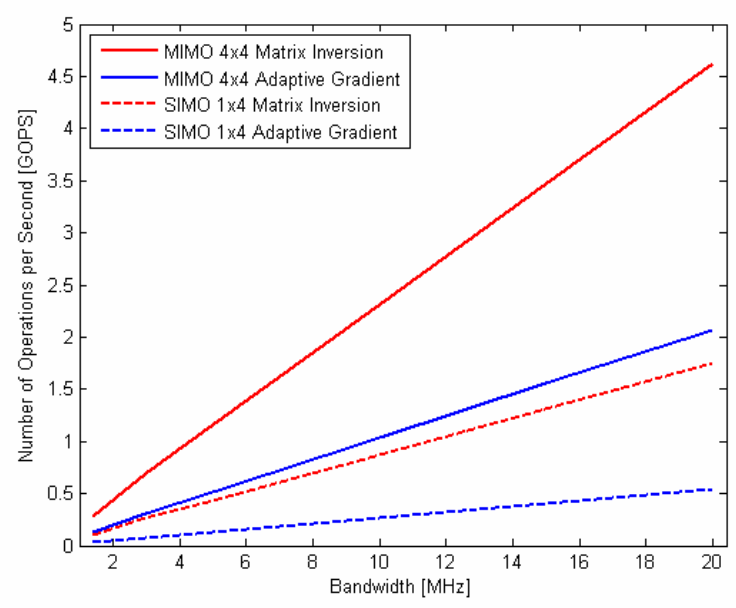

Figure 4. Number of operations as a function of bandwidth allocated to UE.

\section{DISCUSSIONS AND CONCLUSIONS}

In this study we have analyzed performance of linear SUMIMO detector applied in 3GPP LTE wireless systems. We have considered MMSE linear detectors based on (i) explicit matrix inversion, and (ii) adaptive gradient algorithm. We have presented the average achievable data rates as a function of different mobile terminal speeds, using the Jakes model, with symbol-by-symbol temporal variations. Mean and cell-edge rates have been determined using two-dimensional multi-cell model. In addition, the implementation complexity is analyzed.

At the expense of higher implementation complexity, for higher mobile terminal speeds and SINR the detector based on explicit matrix inversion has been shown to outperform the one based on the adaptive gradient algorithm. Furthermore, we have showed that the corresponding SIMO solution outperforms MIMO for moderate and high mobile terminal speeds. Significant gains of MIMO with linear detection over SIMO are present only in a very limited number of channel instantiations. Based on the presented results, the MIMO transmission should be applied only at low mobile terminal speeds, high SINR, and with the MIMO detector using explicit matrix inversion. At moderate and high speeds, a single layer SIMO transmission should be applied, since it outperforms MIMO alternatives both in terms of mean and cell-edge rates.

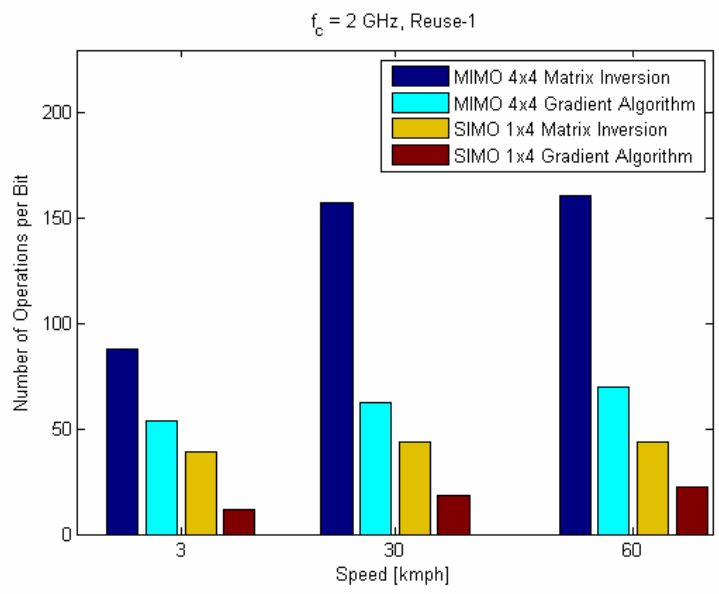

Figure 5. Ratio between the total number of operations versus mean rate.

Complexity-wise, the SIMO schemes have been shown to be very effective because they require the lowest number of operations per transmitted information bit. The SIMO detector based on the adaptive gradient algorithm is particularly effective.

Since there is a significant performance gap between the idealized MIMO capacity and the achievable rates of the linear MIMO detectors, we expect that non-linear schemes may provide some additional improvements [10]. Therefore, our future work will focus on non-linear MIMO detectors, addressing their performance and implementation complexity.

\section{REFERENCES}

[1] E. Dahlman, S. Parkvall, J. Sköld, P. Beming, $3 G$ Evolution - HSPA and LTE for Mobile Broadband. $2^{\text {nd }}$ Edition, Academic Press, 2008.

[2] F. Khan, LTE for $4 G$ Mobile Broadband - Air Interface Technologies and Performance. Cambridge University Press, 2009.

[3] 3GPP TS 36.401 Evolved Universal Terrestrial Radio Access Network (E-UTRAN): Architecture Description.

[4] 3GPP TS 36.201 Evolved Universal Terrestrial Radio Access (EUTRA): Long Term Evolution (LTE) Physical Layer General Description.

[5] 3GPP TS 36.211 Evolved Universal Terrestrial Radio Access (EUTRA): Physical Channels and Modulation.

[6] S. Verdu, Multiuser Detection. Cambridge University Press, 1998.

[7] G. H. Golub and C. F. Van Loan, Matrix Computations. Johns Hopkins University Press, $3^{\text {rd }}$ Edition, 1996.

[8] S. Hykin, Adaptive Filtering. $2^{\text {nd }}$ Edition, Prentice Hall, 1991.

[9] W. Jakes, Microwave Mobile Communications. John Wiley \& Sons, 1974.

[10] B. M. Hochwald and S. ten Brink, "Achieving Near-Capacity On a Multiple-Antenna Channel," IEEE Transactions on Information Theory, vol. 51, pp. 389-399, March 2003. 